EGU21-15471, updated on 13 Sep 2021

https://doi.org/10.5194/egusphere-egu21-15471

EGU General Assembly 2021

(c) Author(s) 2021. This work is distributed under

the Creative Commons Attribution 4.0 License.

\title{
Frequency-domain electromagnetic induction inversion with randomized tensor decomposition
}

\author{
João Narciso ${ }^{1}$, Mingliang Liu ${ }^{2}$, Ellen Van De Vijver ${ }^{3}$, Leonardo Azevedo ${ }^{1}$, and Dario Grana ${ }^{2}$ \\ ${ }^{1}$ CERENA - Centro de Recursos Naturais e Ambiente, DECivil, Instituto Superior Técnico, Universidade de Lisboa, Lisboa, \\ Portugal (joao.narciso@tecnico.ulisboa.pt) \\ ${ }^{2}$ Department of Geology and Geophysics, School of Energy Resources, University of Wyoming, United States \\ ${ }^{3}$ Department of Environment, Ghent University, Gent, Belgium
}

Near-surface systems can be complex and highly heterogeneous. The complex nature of these systems makes their numerical modelling a challenging problem in geosciences. Geophysical survey methods combined with direct measurements have been widely used to characterize the spatial distribution of the near-surface physical properties. Within this scope, geophysical inversion has been a preferable tool to predict quantitively the spatial distribution of the relevant near-surface properties. Ensemble-based data assimilation techniques are common geophysical inversion methods used in problems related to subsurface modelling and characterization. These methods allow the accurate prediction of the spatial distribution of the subsurface properties and have the ability to assess the uncertainty about the model predictions. However, these are computationally demanding inversion techniques, which makes their applicability to large data sets prohibitive.

This study presents the application of a computationally efficient ensemble-based data assimilation technique for inversion of a large-scale frequency-domain electromagnetic induction survey data set. The inversion method is based on randomized high-order singular value decomposition. We combine randomized linear algebra with high-order singular value decomposition, which allows to perform data assimilation in a low-dimensional model and data space. This inversion approach satisfies two objectives: it reduces the computational burden of the inversion and has the same characteristics as conventional ensemble-based data assimilation methods. The inversion method presented herein predicts the spatial distribution of subsurface electrical conductivity and magnetic susceptibility from frequency-domain electromagnetic induction data (as related to the in-phase and quadrature FDEM signal components).

The method is illustrated in a three-dimensional real case application where a set of geophysical and borehole data is available. The log-set composed by electrical conductivity and magnetic susceptibility is used as conditioning data to generate a prior ensemble of numerical threedimensional models with geostatistical simulation. The predicted posterior distribution generates synthetic frequency-domain electromagnetic induction data that reproduces the observed data. The model predictions at a blind well location, not used in the generation of the prior ensemble, agree will with the observed log data, validating the quality of the applied method. 
